\title{
A modified index for the description of the ionospheric short- and long-term activity
}

\author{
J. Mielich and J. Bremer \\ Leibniz-Institute of Atmospheric Physics at the Rostock University, 18225 Kühlungsborn, Schloss-Str. 6, Germany
}

Received: 23 September 2010 - Revised: 24 November 2010 - Accepted: 9 December 2010 - Published: 21 December 2010

\begin{abstract}
A modified ionospheric activity index AI has been developed on the basis of ionospheric $f o \mathrm{~F} 2$ observations. Such index can be helpful for an interested user to get information about the current state of the ionosphere. Using ionosonde data of the station Juliusruh $\left(54.6^{\circ} \mathrm{N} ; 13.4^{\circ} \mathrm{E}\right)$ this index has been tested for the time interval from January 1996 until December 2008. This index has no diurnal and seasonal variations, only a small positive dependence on the solar activity could be found. The variability of this index has, however, a marked seasonal variability with maxima during the equinoxes, a clear minimum in summer, and enhanced values in winter. The observed variability of AI is strongly correlated with the geomagnetic activity, most markedly during the equinoxes, whereas the influence of the solar activity is markedly smaller and mostly insignificant. Strong geomagnetic disturbances cause in middle latitudes in general negative disturbances in $\mathrm{AI}$, mostly pronounced during equinoxes and summer and only partly during winter, thus in agreement with the current physical knowledge about ionospheric storms.
\end{abstract}

Keywords. Radio science (Ionospheric physics)

\section{Introduction}

In the past solar activity indices (e.g. solar sunspot number $\mathrm{R}$ or solar radio flux at $10.7 \mathrm{~cm}, \mathrm{~F} 107)$ have been used for ionospheric investigations. Later also ionospheric activity indices were deduced from ionosonde observations (Minnis, 1955; Minnis and Bazzard, 1959, 1960). However, these indices are only monthly values and therefore mainly helpful for long-term investigations.
In the framework of the EC eContent programme, the Digital upper Atmosphere Server (DIAS) project has created a computational infrastructure for a European digital data collection describing the state of the ionospheric part of the upper atmosphere over Europe (Belehaki et al., 2005). One component of this project deals with the creation of ionospheric activity indices (AI) which describe the current ionospheric state on an hourly or even shorter time basis. These indices have been derived from ionosonde observations of the ionospheric F2-layer, mainly by use of $f o \mathrm{~F} 2$ and $\mathrm{M}(3000) \mathrm{F} 2$ values. Such ionospheric activity indices are important for users to know if the current ionospheric state is that to be expected from mean solar activity level or is modified by short term solar, geomagnetic or atmospheric disturbances (solar flares, geomagnetic events, atmospheric waves etc.). Details of this procedure and first results have been reported by Bremer et al. (2006).

In the presented paper these indices are slightly modified to remove a small bias caused by seasonal variations of the ionospheric parameters. Whereas Bremer et al. (2006) investigated data of a short time interval (October 2003 until March 2005) at different European ionosonde stations here long-lasting data during the solar activity cycle 23 (January 1996 until December 2008) are used from observations at the ionosonde station Juliusruh $\left(54.6^{\circ} \mathrm{N} ; 13.4^{\circ} \mathrm{E}\right)$. Besides hourly AI values have been derived based only on hourly $f o \mathrm{~F} 2$ observations as these indices are more sensitive to ionospheric variability than the indices derived from M(3000)F2 values.

In Sect. 2 the developed method and the used data are described whereas the main experimental results are presented in Sect. 3 followed by a discussion of the results (Sect. 4) and some general conclusions (Sect. 5). 


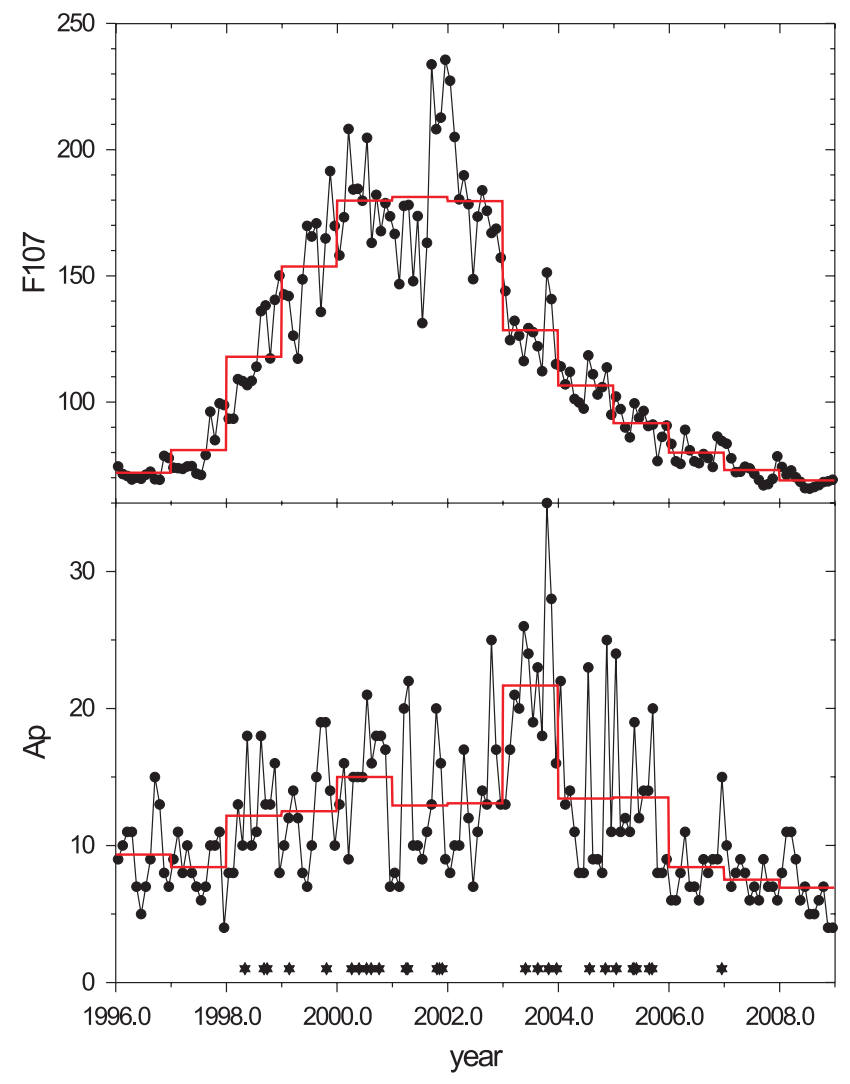

Fig. 1. Variation of the solar activity index F107 (upper part) and of the planetary geomagnetic activity index Ap (lower part), both based on monthly mean values (black) and yearly mean values (red). Geomagnetic disturbances with daily values $A p \geq 80$ are marked in the lower part by black asterisks.

\section{Description of the experimental data and methodical investigations}

In the analyses presented in this paper hourly $f o \mathrm{~F} 2$ values of the station Juliusruh (URSI code JR055) have been used for the time interval from January 1996 until December 2008, thus including the whole solar cycle 23 . In Fig. 1 the solar and geomagnetic activity variations during this period are presented by monthly mean values of the solar $10.7 \mathrm{~cm}$ radio wave radiation (F107) in the upper part of this figure and of the planetary geomagnetic activity index Ap in the lower part. In addition yearly mean values of both indices are presented by red step like curves. Thus, years with low solar activity (e.g. 1996, 2007-2008) as well as high solar activity (2000-2002) are included in the chosen interval. The geomagnetic variation is more fluctuating with maximum values during the descending part of the solar cycle (maximum values in 2003). In the lower part of Fig. 1 also strong geomagnetic disturbances with daily geomagnetic activity values $A p \geq 80$ are designated by black asterisks.

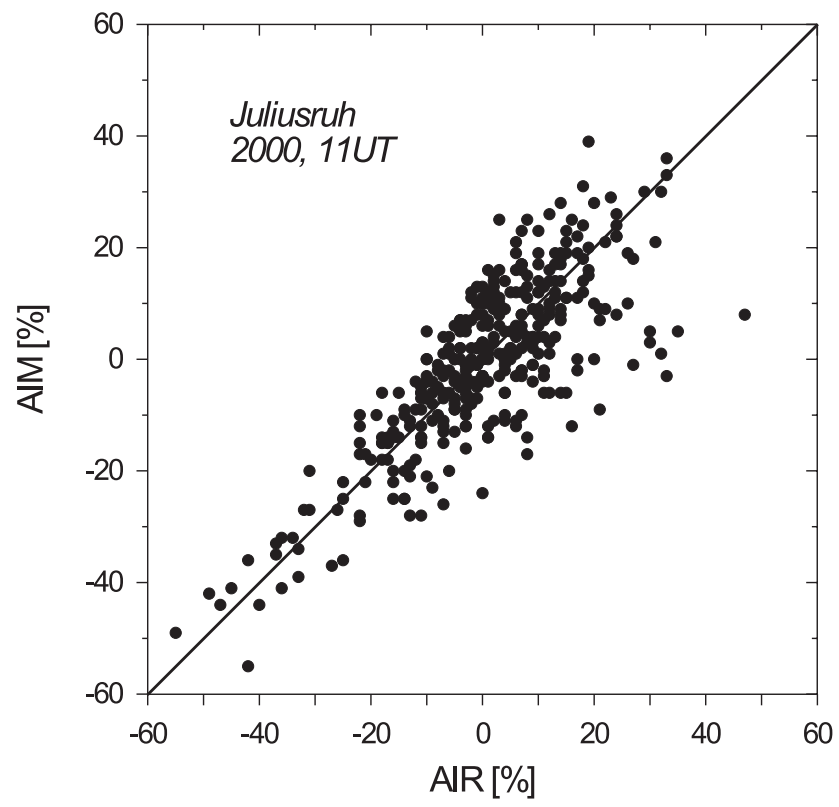

Fig. 2. Comparison of hourly AI values derived by different reference values (AIM: based on median values, AIR: based on regression values) using experimental ionosonde data observed in Juliusruh at 11:00 UT during the year 2000 .

In this paper only hourly $f o \mathrm{~F} 2$ values have been used for the derivation of AI values, such indices can of course also be derived for data at shorter intervals (e.g. ionosonde data measured every $15 \mathrm{~min}$ ). Here we used manually corrected $f o \mathrm{~F} 2$ data to eliminate possible incorrect values. For real time analyses of the ionospheric activity, however, automatically scaled ionosonde data have to be used for the estimation of the ionospheric activity indices.

The ionospheric activity index $\mathrm{AI}$ in per cent is derived by comparison of the current $f o \mathrm{~F} 2$ values with an undisturbed reference value $f o \mathrm{~F}^{\text {ref }}$ according to the following simple formula:

$\mathrm{AI}(f o \mathrm{~F} 2)=100\left(f o \mathrm{~F} 2-f o{ }^{\mathrm{ref}} 2^{\mathrm{ref}}\right) / f_{o} \mathrm{~F} 2^{\mathrm{ref}}$

In Bremer et al. (2006) the reference value was the median value of the last 30 (or 27) days before the current $f o \mathrm{~F} 2$ value at the same local time. In this paper we use a slightly modified procedure, calculating the reference value by a simple linear regression analysis with the data of the last 27 days before the current $f o \mathrm{~F} 2$ value and calculate $f o \mathrm{~F} 2{ }^{\text {ref }}$ from this regression equation for the same hour as the current value $f o \mathrm{~F} 2$. The choice of a 27 days interval is physically more plausible due to the solar rotation period. In practice, however, it is not essential if the interval is 27 or 30 days as demonstrated in Bremer et al. (2006).

In principle it is also possible to derive AI values also from $\mathrm{M}(3000) \mathrm{F} 2$ observations. The advantage is that such values contain information about ionospheric height changes. However, as shown in Bremer et al. (2006) these activity indices 


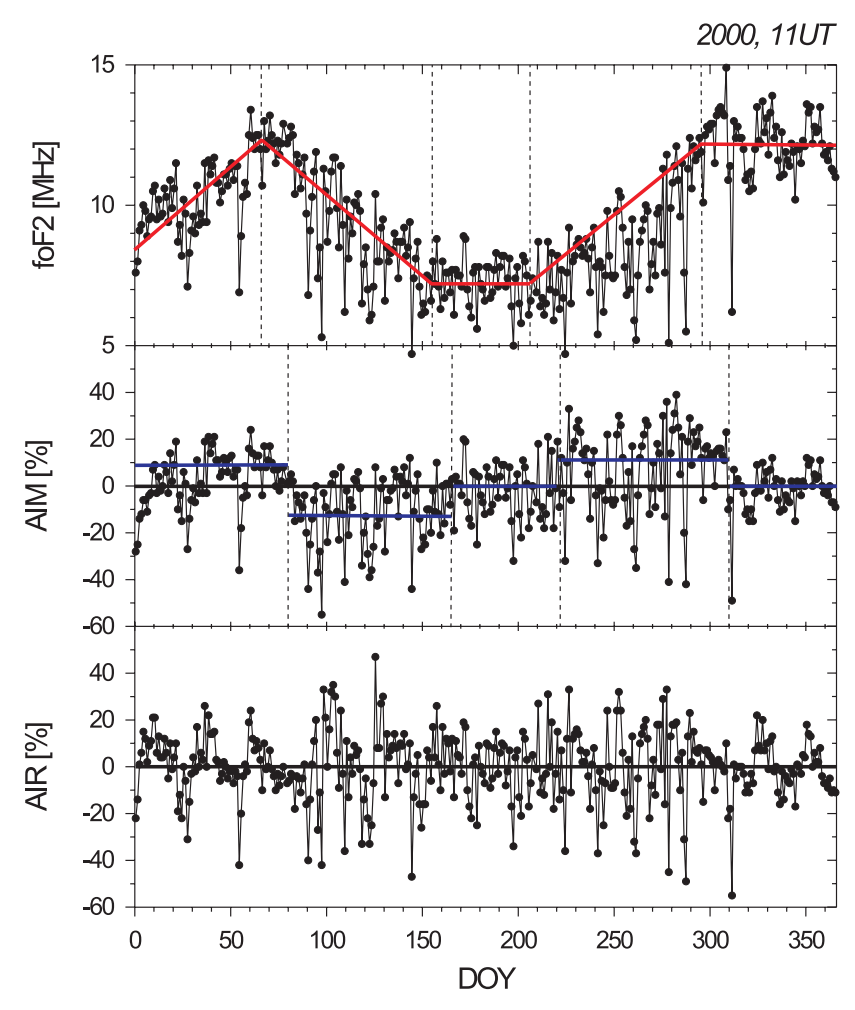

Fig. 3. Seasonal variation of $f o F 2$ (upper part), AIM values (middle part), and AIR (lower part) from ionosonde data of Juliusruh at 11:00 UT during the year 2000. The red and blue lines represent the mean variations of the different parameters within different time intervals.

are less sensitive to ionospheric variability. Therefore, we concentrate in this paper only on investigations of AI values derived from $f o \mathrm{~F} 2$ observations according to Eq. (1).

In Fig. 2 an example is shown using hourly AI values at 11:00 UT during the year 2000 for the comparison of AI values derived with different reference values (AIM: using median values; AIR: using regression analysis values). As to be seen, there is a marked scatter around the ideal $45^{\circ}$-line. The reason of this scatter may an incorrect estimation of some reference values. In Fig. 3 the same values as used in Fig. 2 are presented in dependence on season. In the upper part of Fig. 3 the hourly $f o \mathrm{~F} 2$ values are drawn, characterized by a marked seasonal variation with some parts of increasing, decreasing, and nearly constant values. These intervals are separated by vertical dashed lines and the $f o \mathrm{~F} 2$ variations within these intervals are schematically adapted by linear red lines. These lines are only for demonstration of the general tendencies within the intervals, they are not accurately calculated. In the middle part of Fig. 3 AIM values are presented with different mean values within intervals which are shifted by about 14 days concerning to the above defined $f o \mathrm{~F} 2$ intervals. For positive $f o \mathrm{~F} 2$ trends the corresponding AIM mean values are positive, for negative $f o \mathrm{~F} 2$ trends the AIM mean values are negative, and for zero $f o \mathrm{~F} 2$ trends also the AIM mean val-

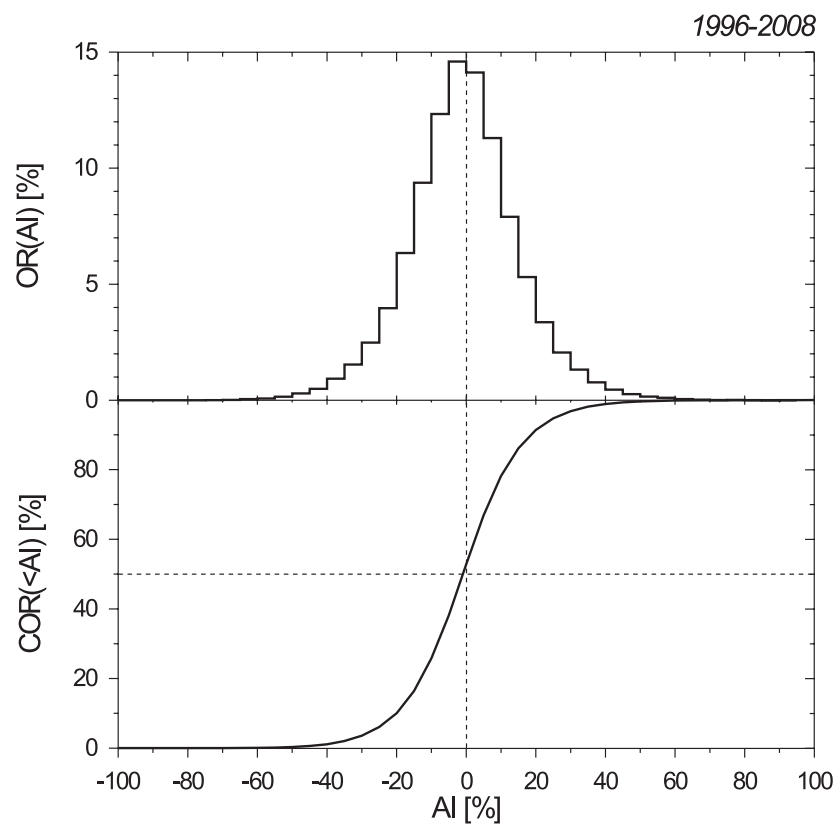

Fig. 4. Histogram of hourly AI values derived from occurrence rates OR(AI) of width 5\% for the interval between 1996 and 2008 (upper part) and the corresponding cumulative occurrence rate $\mathrm{COR}(<\mathrm{AI})$ (lower part).

ues vary around the zero level. These mean AIM values are schematically characterized by horizontal blue lines. The explanation of this phenomenon is very simple. The reference value $f o \mathrm{~F} 2^{\text {ref }}$ is too low if its median value is estimated from foF 2 data with a positive seasonal trend and therefore the corresponding AIM value is too large. Contrary the $f o \mathrm{~F}^{\text {ref }}$ values are too big if the $f o \mathrm{~F} 2$ trends are negative and therefore the resulting AIM values are too small. Only if there are no $f o \mathrm{~F} 2$ trends the estimated $f o \mathrm{~F} 2^{\text {ref }}$ values and the derived AIM values are correct. The time shift between the AIM intervals and the $f o \mathrm{~F} 2$ intervals (marked by the different vertical dashed lines in AIM compared with $f o \mathrm{~F} 2$ panel) is caused by the mean time difference between the current $f o \mathrm{~F} 2$ values and the centre of the 27 days reference interval.

Using the procedure with the estimation of the reference values $f o 2_{2}{ }^{\text {ref }}$ by a regression analysis such problems do not exist as to be seen in the lower part of Fig. 3 where the corresponding AIR values are presented for the same data set as used in the other parts of Fig. 3. Here no marked seasonal discontinuities can be detected. Therefore, in the remaining part of this paper only AIR values have been used, but for simplification they are called now AI.

\section{Experimental results}

To demonstrate the variability of the AI values, in the upper part of Fig. 4 a histogram is shown for all hourly AI values estimated during the time interval from 1996 until 2008 


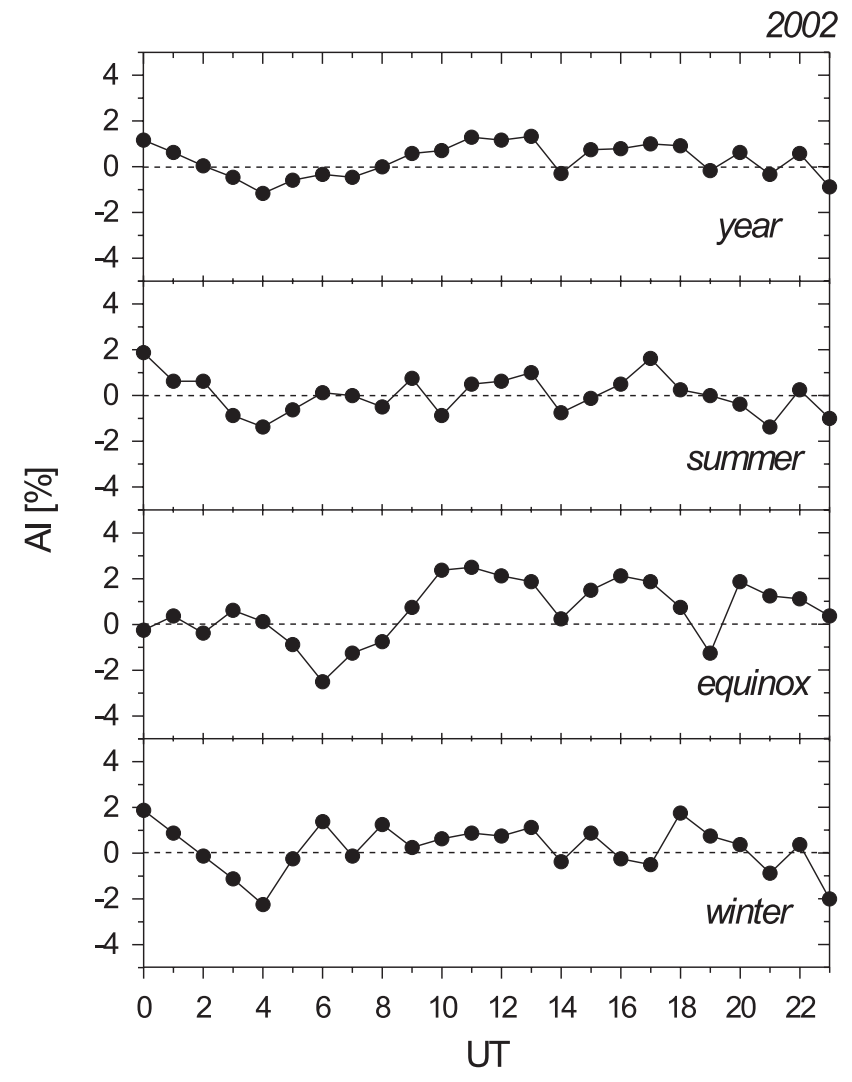

Fig. 5. Diurnal variations of mean AI values for the full year 2002 and different seasons of this year (summer, equinox, and winter).

from ionosonde observations in Juliusruh. Here $\mathrm{OR}(\mathrm{AI})$ is the occurrence rate of $\mathrm{AI}$ for intervals of width $5 \%$. In the lower part of Fig. 4 the corresponding cumulative occurrence rate $\mathrm{COR}(<\mathrm{AI})$ is presented. As to be seen from the upper and lower parts of Fig. 4 the distribution of the AI values is symmetric with regard to the occurrence rate maximum at AI values near zero.

\subsection{Diurnal variation of AI values}

On the basis of monthly median values of hourly AI data during the year 2002 their mean diurnal variations have been derived for the full year and different seasons (summer, equinox, and winter). The results are presented in Fig. 5. In all cases no distinct diurnal variation can be seen. The hourly mean values are near zero (dashed lines) or slightly positive.

In Fig. 6 mean diurnal variations of AI are presented for different years (1996, 2002, and 2008) and for the full observation interval between 1996 and 2008. In agreement with Fig. 5 also in Fig. 6 no market diurnal variations can be detected. However, the diurnal mean values (identical with yearly mean values) are not always near zero. During years of low solar activity $(1996,2008)$ these mean values are negative and during high solar activity (2002)

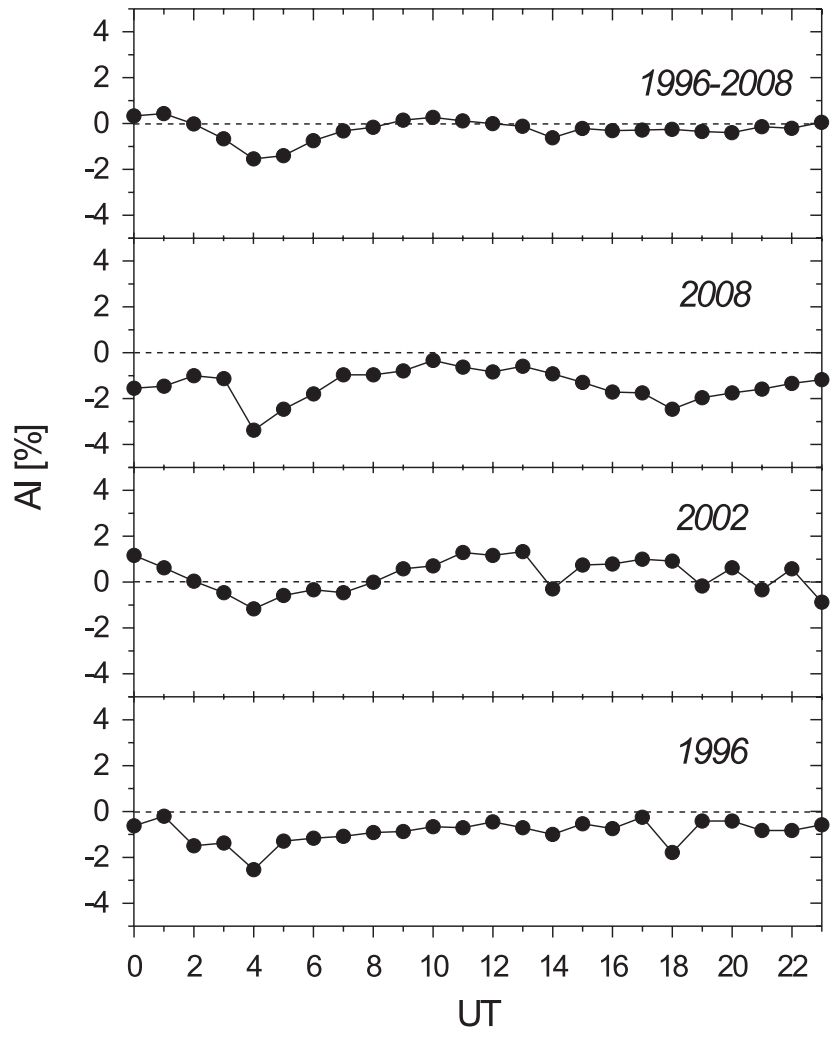

Fig. 6. Diurnal variations of mean AI values for different years (1996, 2002, and 2008) and for the full interval between 1996 and 2008.
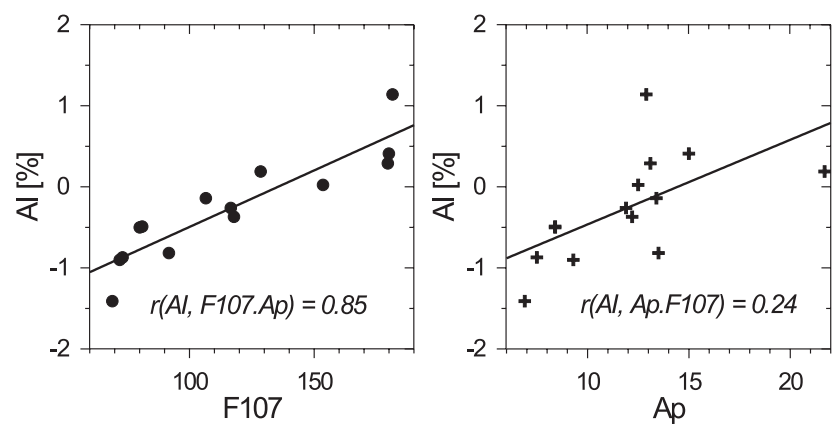

Fig. 7. Dependence of yearly mean AI values on solar activity (left part) and on geomagnetic activity (right part). The included values are the corresponding partial correlation coefficients.

slightly positive. To investigate this phenomenon in more detail, the yearly mean values have been estimated for all years between 1996 and 2008. In Fig. 7 these data are shown in dependence on solar activity (F107, left part) and on geomagnetic activity (Ap, right part). In both cases a significant correlation seems to exist. As, however, F107 and Ap are correlated, partial correlation coefficients have to be estimated. These values are included in both parts of Fig. 7. Whereas the partial correlation coefficient $r$ (AI, 


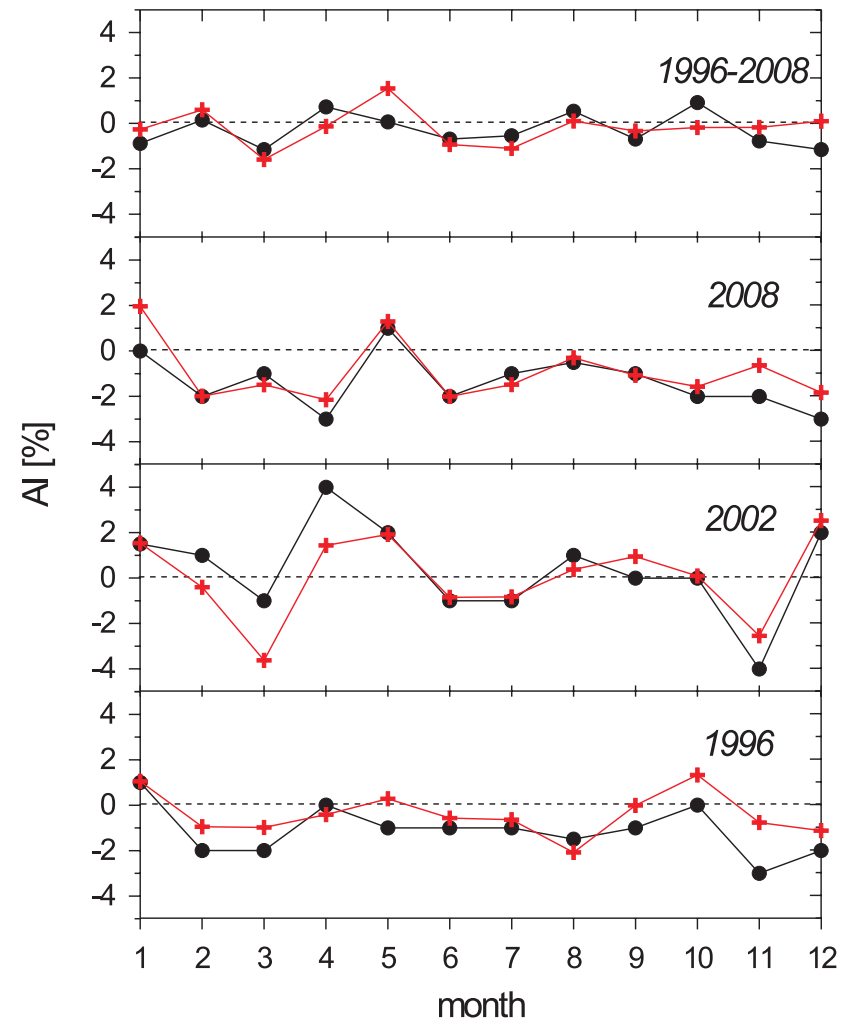

Fig. 8. Seasonal variations of mean AI values for different years (1996, 2002, and 2008) and for the full interval between 1996 and 2008 using monthly mean (red) or median values (black).

F107.Ap) $=0.85$ is statistically significant with $>99 \%$, the other correlation $r(\mathrm{AI}, \mathrm{Ap} . \mathrm{F} 107)=0.24$ is insignificant (significance tests according to Taubenheim, 1969). Therefore, the yearly mean values of AI are significantly controlled by the solar activity. The deviations of the yearly mean values from zero are only small (mostly smaller than $\pm 1 \%$ ) as to be seen from Fig. 7.

\subsection{Seasonal variation of AI values}

From hourly AI values monthly median as well as monthly mean values have been calculated for different years and for the full period between 1996 and 2008. The results are presented in Fig. 8 (median values in black, mean values in red). In all cases no marked seasonal variation can be seen. Only a small influence of the solar activity (F107) can be detected between the individual years in agreement with the results presented in Fig. 7.

In Fig. 9 additional results concerning the seasonal variation are presented on the basis of all hourly AI values during the time interval between 1996 and 2008. In part (b) of Fig. 9 the variation of the upper (uq) and lower quartiles (lq) of the hourly AI values are shown. From these data the quartile difference dq is derived and presented in part (a) together with the standard deviation (STD, red curve). Both data sets

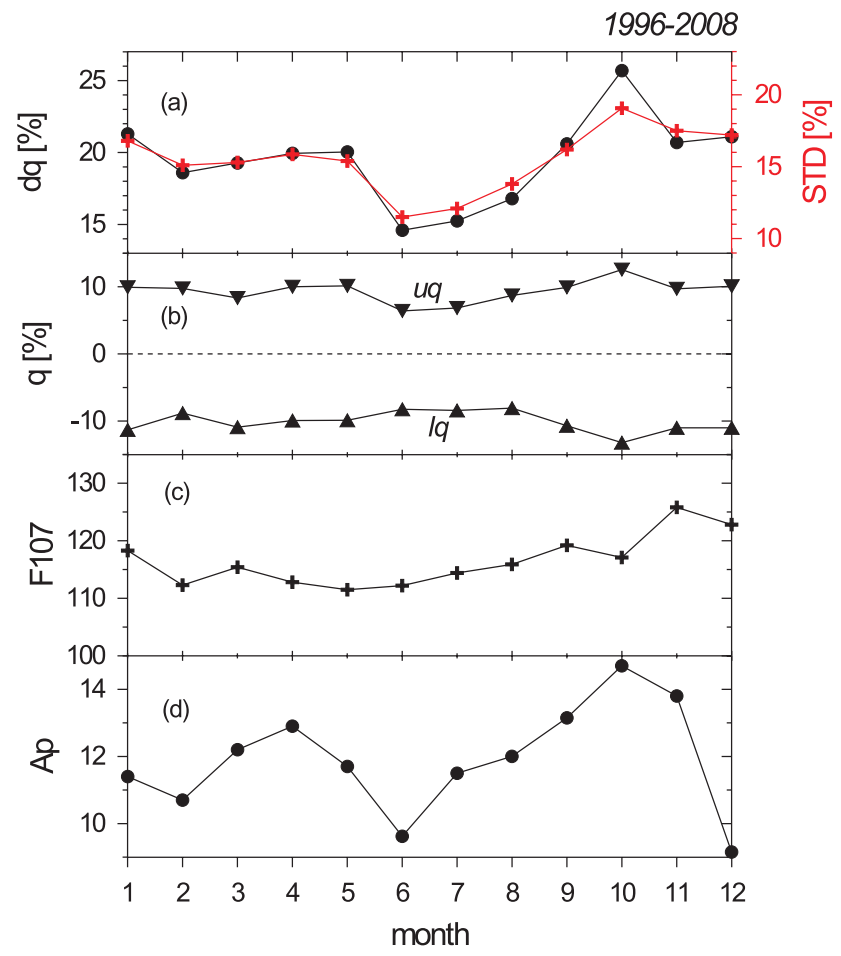

Fig. 9. Seasonal variation of (a) the quartile difference dq (black) and the standard deviation STD (red) as well as (b) the upper and lower quartiles (uq and lq) basing on hourly AI values. The corresponding variations of the solar and geomagnetic activity are shown in (c) and (d). All data were derived for the period between 1996 and 2008 .

of dq and STD, describing the variability of the AI data, have nearly identical seasonal variations with maxima near the equinoxes (most pronounced in autumn), a marked minimum in summer (June, July) and enhanced values in winter. Most parts of these seasonal variations are closely connected with the mean geomagnetic activity variation (d), especially the equinoctial maxima (also here the maximum in autumn dominates) and the minimum in summer show strong similarities. The small variation of the solar activity in part (c) with slightly enhanced F107 values during winter is unimportant for the explanation of the AI variability parameters in parts (a) and (b) of Fig. 9. The small seasonal variation of F107 is caused by the seasonally changing distance between sun and Earth. Therefore, the observed solar radio flux F107 is about $7 \%$ stronger in winter than in summer.

The seasonal variations of the cumulative occurrence rates $\mathrm{COR}$ are estimated for positive $\mathrm{AI}$ by $\mathrm{COR}(\mathrm{AI}>X)$ and for negative $\mathrm{AI}$ by $\mathrm{COR}(\mathrm{AI}<-X)$. In the lower part of Fig. 10 these values are shown for three different levels $X=10,20$, and 30 by black curves for negative AI and by red curves for positive AI basing on all data between 1996 and 2008 . In the middle and upper parts of Fig. 10 the seasonal variations of the cumulative occurrence rate are separately presented for low $\left(\mathrm{F} 107_{\min }\right)$ and high solar activity $\left(\mathrm{F} 107_{\max }\right)$. 


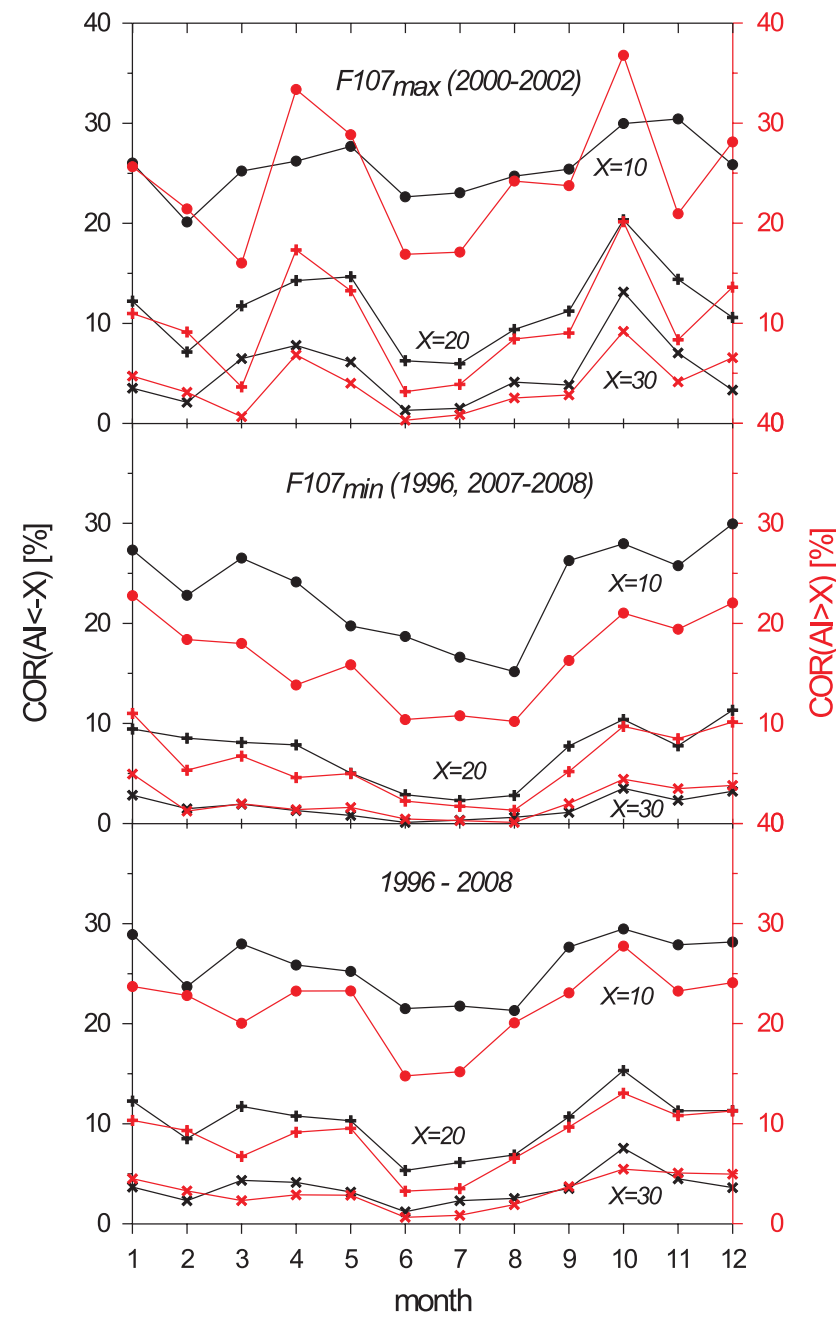

Fig. 10. Seasonal variation of the cumulative occurrence rate for negative (black) and positive hourly AI values (red) for different threshold values X separately for the full time interval (lower part), years of low (middle part) and high solar activity (upper part).
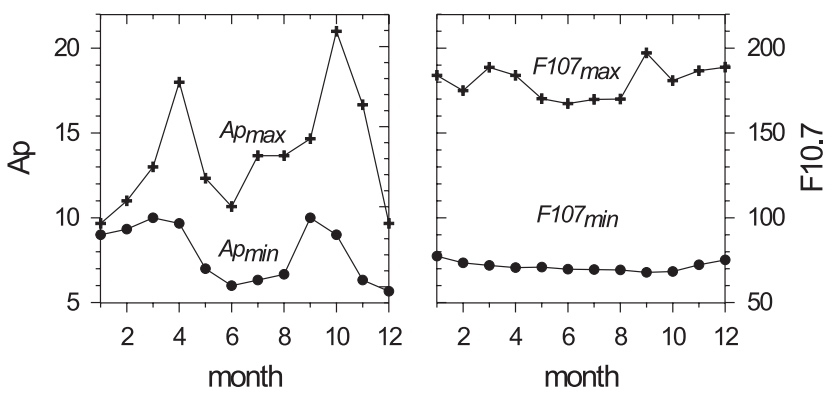

Fig. 11. Seasonal variation of the geomagnetic (left part) and solar activity (right part) for years of low (1996, 2007-2008) and high solar activity (2000-2002).

In all cases the cumulative occurrence rates are characterized by equinoctial maxima, a pronounced minimum in summer (June, July), and relative strong values in winter. In most

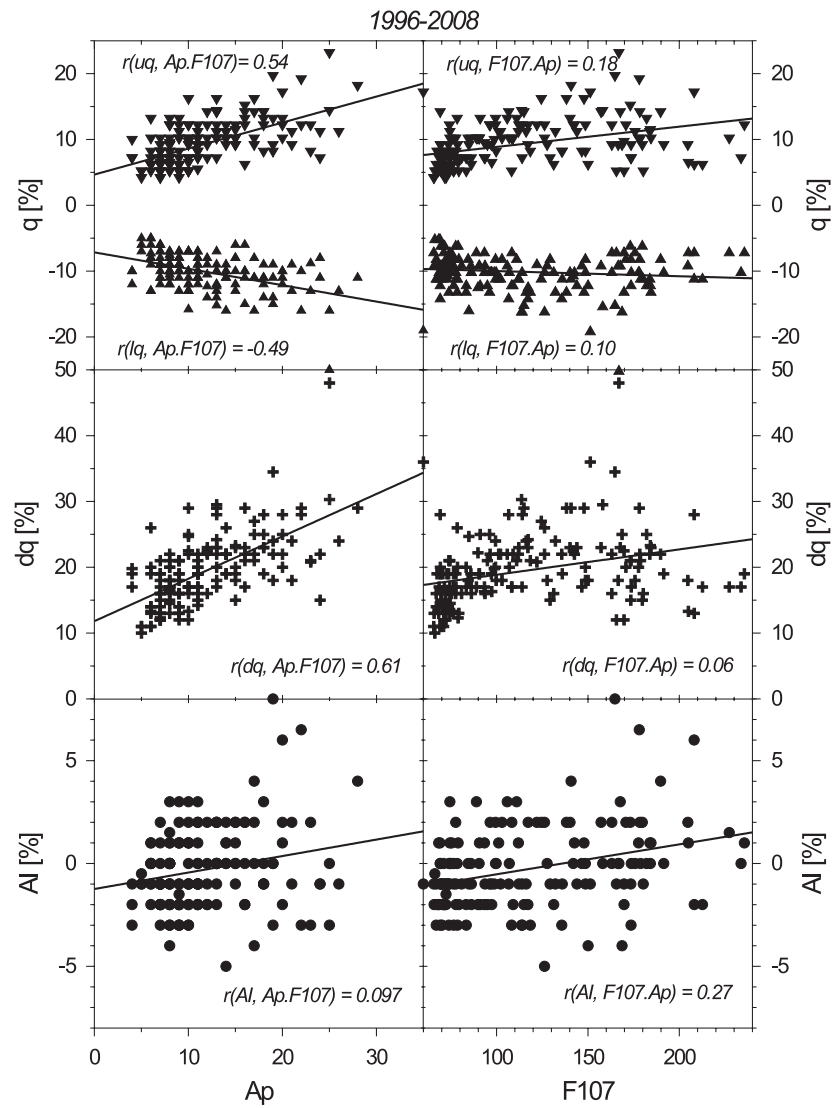

Fig. 12. Dependence of monthly upper quartile uq, lower quartile lq, quartile difference dq, and AI median values on geomagnetic activity (left part) and on solar activity (right part). The black lines are simple regression lines whereas the included values are partial correlation coefficients.

cases the occurrence rate for negative AI values is higher than for positive values. The equinoctial maxima are most pronounced during years of high solar activity, and these maxima are stronger during autumn than during spring. These features of the seasonal variations of the COR values are strongly connected with the variation of the geomagnetic activity as to be seen in Fig. 11. The equinoctial maxima of Ap are stronger at years of high solar activity. Furthermore the autumnal maximum is more pronounced than the vernal maximum. The influence of the solar activity changes on the cumulative occurrence rates should, however, be small as already discussed above in connection with Fig. 7.

\subsection{Solar and geomagnetic control of AI values}

In the foregoing Sect. 3.2 there are some indications of a possible geomagnetic and solar control of the variability of the ionospheric activity index AI. Therefore, in this section the results of some additional correlation analyses will be presented. In Fig. 12 monthly median values of the full observation period during 1996 until 2008 are used and their dependence on the geomagnetic Ap index (left 
Table 1. Geomagnetic disturbances with Ap $\geq 80$ during the time interval between 1996 and 2008 .

\begin{tabular}{|c|c|c|c|c|c|}
\hline \multicolumn{2}{|l|}{ summer } & \multicolumn{2}{|l|}{ winter } & \multicolumn{2}{|l|}{ equinox } \\
\hline date & Ap & date & Ap & date & Ap \\
\hline 4 May 1998 & 101 & 18 February 1999 & 80 & 25 September 1998 & 117 \\
\hline 27 August 1998 & 144 & 6 November 2001 & 142 & 22 October 1999 & 91 \\
\hline 24 May 2000 & 93 & 24 November 2001 & 104 & 6 April 2000 & 82 \\
\hline 15 July 2000 & 164 & 20 November 2003 & 150 & 5 October 2000 & 116 \\
\hline 12 August 2000 & 123 & 10 November 2004 & 161 & 31 March 2001 & 192 \\
\hline 29 May 2003 & 109 & 18 January 2005 & 84 & 11 April 2001 & 85 \\
\hline 18 August 2003 & 108 & 15 December 2006 & 94 & 22 October 2001 & 96 \\
\hline 27 July 2004 & 186 & & & 29 October 2003 & 204 \\
\hline 8 May 2005 & 91 & & & 11 September 2005 & 101 \\
\hline 15 May 2005 & 87 & & & & \\
\hline 30 May 2005 & 90 & & & & \\
\hline 24 August 2005 & 102 & & & & \\
\hline
\end{tabular}

part) and on the solar activity index F10.7 (right part) investigated. Here we employed the monthly median values of AI (lower part), of the monthly upper and lower quartiles uq and lq (upper part) as well as the quartile difference $\mathrm{dq}=\mathrm{uq}-\mathrm{lq}$. The straight lines are the simple linear regression lines. Furthermore in each field correlation values are included. Due to the correlation between the solar and geomagnetic activity here again partial correlation coefficients have been estimated: $r(\mathrm{X}, \mathrm{Ap} . \mathrm{F} 107)$ and $r(\mathrm{X}$, F107.Ap) with $X=$ AI, uq, lq, or dq. The significance levels were derived with normal statistical methods (Student's t-test) according to Taubenheim (1969). Whereas the partial correlation coefficients $r$ (uq, Ap.F107), $r$ (lq, Ap.F107), $r$ (dq, Ap.F107), are highly significant (>99\%), their corresponding coefficients $r$ (uq, F107.Ap), $r$ (lq, F107.Ap), and $r$ (dq, F107.Ap) are all insignificant. Concerning the correlation between AI with F107 and Ap the partial correlation coefficient $r$ (AI, Ap.F107) is insignificant while the correlation $r(\mathrm{AI}, \mathrm{F} 107 . \mathrm{Ap})=0.27$ is significant. However, if monthly mean values are used instead of monthly median values this correlation is slightly smaller with $r(\mathrm{AI}, \mathrm{F} 107 . \mathrm{Ap})=0.17$ (not shown here). Altogether, it can be concluded that there is a small correlation between AI and F107 in agreement with the positive correlation between corresponding yearly mean values as shown in Fig. 7.

In Fig. 13 the quartile differences dq (left part) and the standard deviations STD (right part) of the monthly AI values are drawn in dependence on geomagnetic activity Ap for all values during 1996 until 2008 (upper part) and for different seasons (summer, equinox, winter) in the three further parts. Also here the corresponding partial correlation coefficients $r$ (dq, Ap.F107) and $r$ (STD, Ap.F107) are included in the corresponding parts of the figure. All correlation values are highly significant (>99\%) for both parameters. The correlation at equinoxes is slightly stronger than during the other seasons.

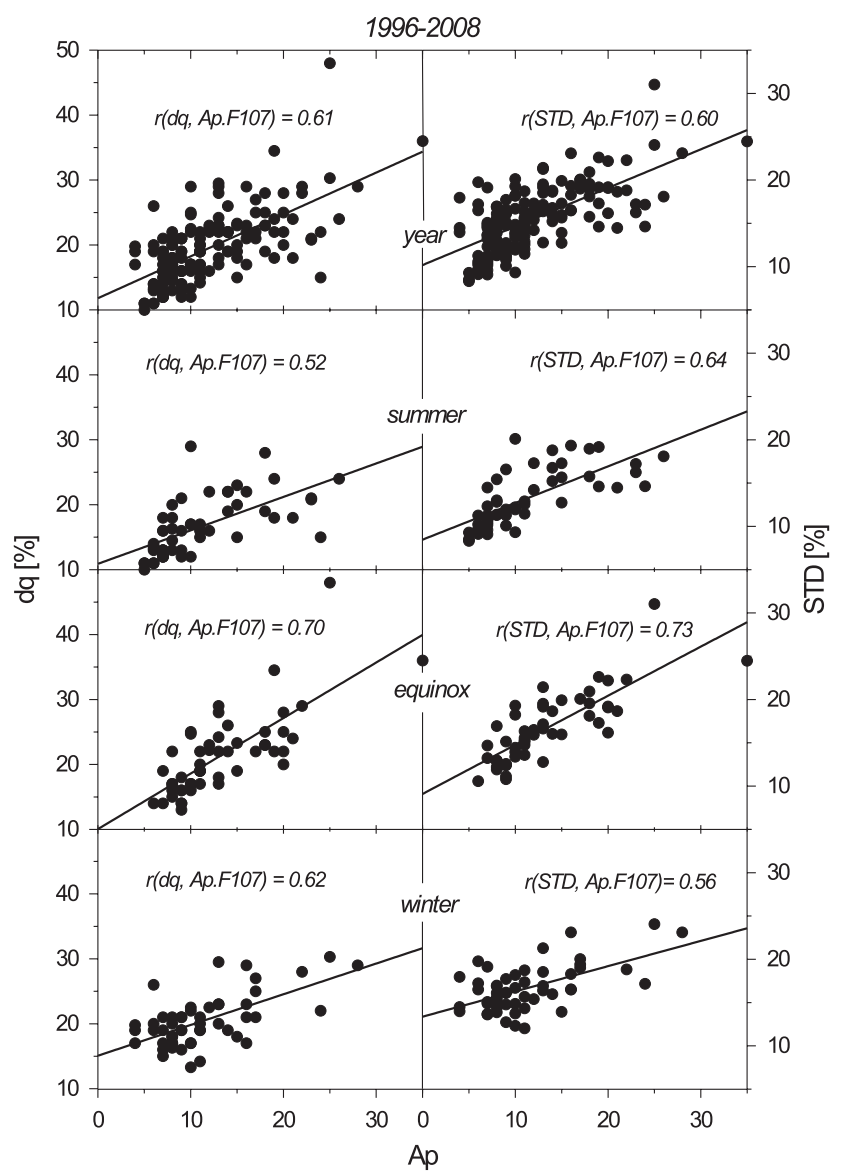

Fig. 13. Dependence of monthly quartile differences dq (left part) and standard deviations STD (right part) on geomagnetic activity for all median values as well as separately for different seasons. The black lines are simple regression lines whereas the included values are partial correlation coefficients. 


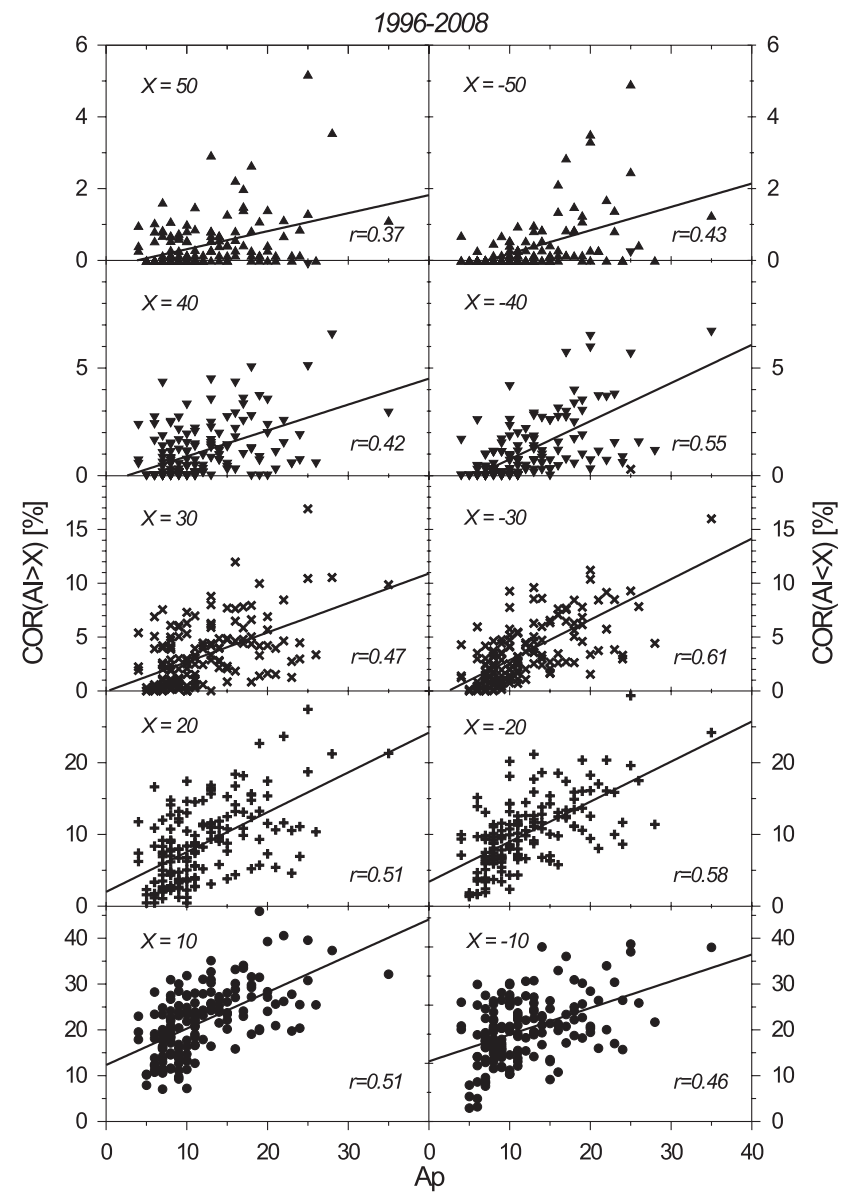

Fig. 14. Dependence of monthly cumulative occurrence rates COR for positive AI values (left part) and negative AI values (right part) on geomagnetic activity for different thresholds X. The black lines are simple regression lines whereas the included values are partial correlation coefficients $\mathrm{r}=\mathrm{r}(\mathrm{COR}(\mathrm{AI}>\mathrm{X})$, Ap.F107) for positive AI or $r=r(\mathrm{COR}(\mathrm{AI}<\mathrm{X})$, Ap.F107) for negative $\mathrm{AI}$ values.

Some correlation analyses have also been made with the cumulative occurrence rate $\mathrm{COR}(\mathrm{AI}>X)$ for different positive $\mathrm{X}$ values as well as with $\mathrm{COR}(\mathrm{AI}<X)$ for negative $\mathrm{X}$ values in dependence on the geomagnetic activity. The results are presented in Fig. 14, again with the corresponding partial correlation coefficients, in the left part for positive AI values and in the right part for negative AI values. All correlation data are highly significant. The correlation values are more pronounced for negative than for positive AI values in nearly all cases.

\subsection{Ionospheric storms}

Investigations of ionospheric storms in connection with geomagnetic disturbances have been carried out for storms during the years 1996 until 2008. We selected geomagnetic disturbances with a maximum daily value of $A p \geq 80$. In Table 1 these days are presented, subdivided in three seasons (sum-

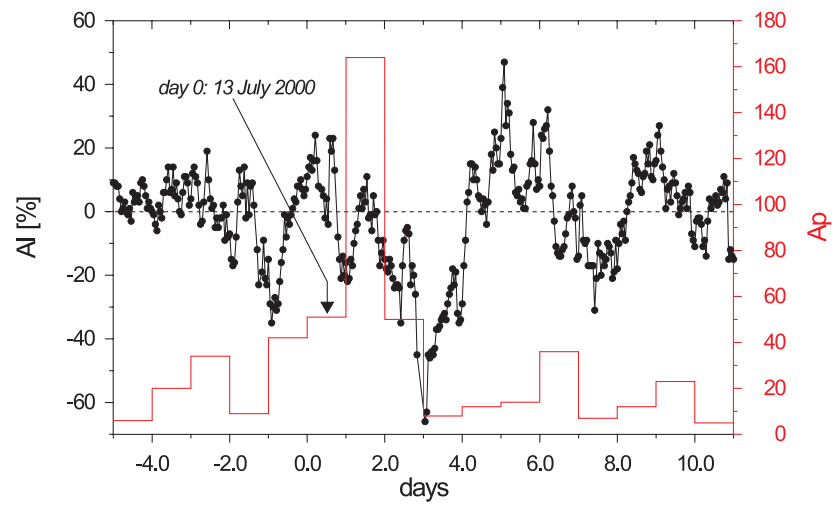

Fig. 15. Variations of hourly AI values in mid-latitudes (black) and daily geomagnetic Ap values (red) in connection with the ionospheric storm in July 2000.

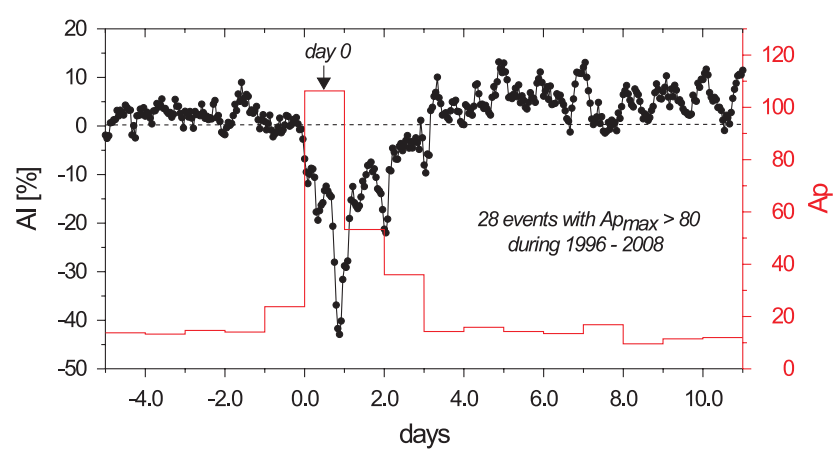

Fig. 16. Mean variation of the hourly AI values (black) and of the daily geomagnetic Ap values (red) derived from 28 individual ionospheric storms with maximum Ap values $\geq 80$ during the time interval from 1996 until 2008 by a superimposed epoch analysis.

mer: May-August, winter: November-February, equinox: March-April and September-October). Normally we used the days with maximum Ap values as key day zero and analysed the interval 5 days before this key day until 10 days after this day. If one or two days before this maximum the disturbances starts with $\mathrm{Ap} \geq 50$, then this day has been chosen as key day. In Fig. 15 such an example is shown with a step like red curve for the daily Ap data and the black curve with hourly AI values. Here the 13 July 2000 with Ap $=51$ is used as key day zero whereas the maximum disturbance with $\mathrm{Ap}=164$ follows one day later. The main ionospheric effect can be detected on days two and three with AI values partly smaller than $-60 \%$.

As to be seen in Table 1, during the chosen time period between 1996 and 200828 events have been detected with the above mentioned Ap criteria. In Fig. 16 the mean AI and Ap variations are shown as the result of a superimposed epoch analysis of these 28 events. Starting with the maximum geomagnetic disturbance on day zero with $\mathrm{Ap}=106$ a negative ionospheric effect begins, achieving its smallest value with $\mathrm{AI}=-43 \%$, and becomes quite undisturbed at day three. 


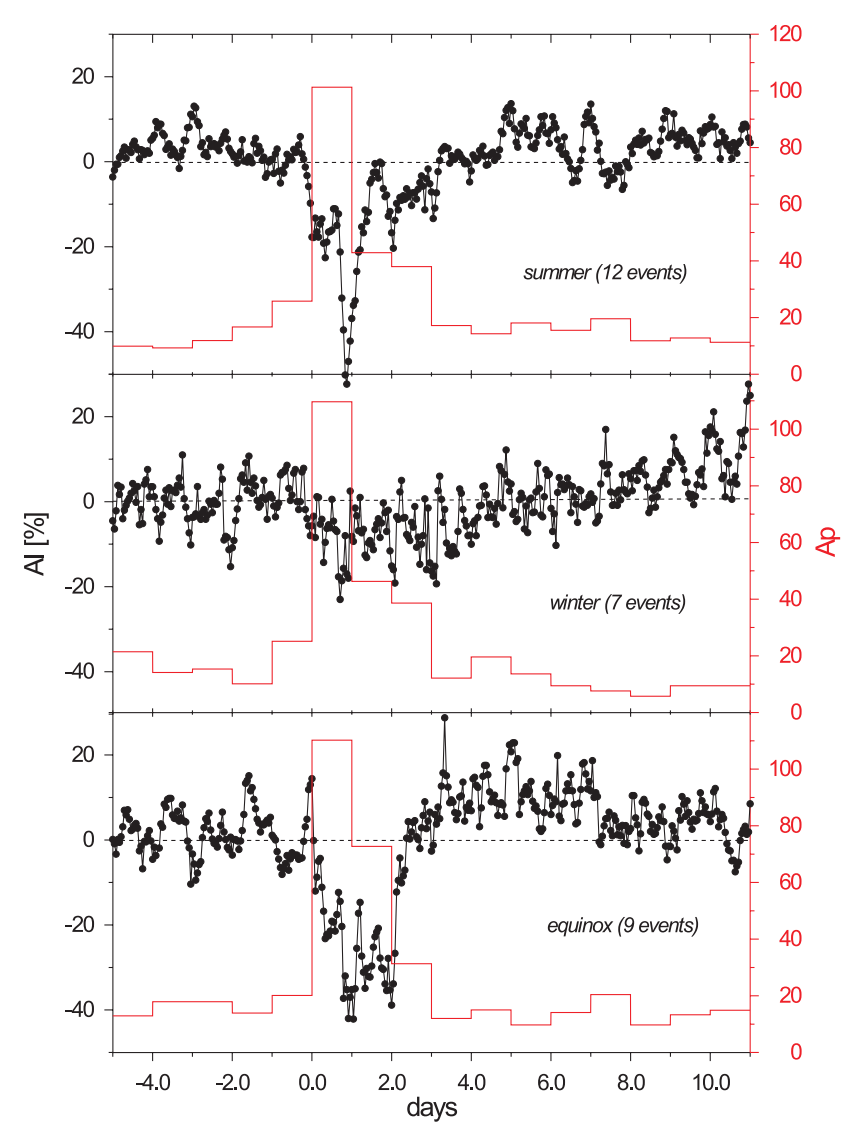

Fig. 17. Mean variation of the hourly AI values (black) and of the daily geomagnetic Ap values (red) derived from 28 individual ionospheric storms with maximum Ap values $\geq 80$ during the time interval from 1996 until 2008 separated for three seasons (summer, winter, equinox) by superimposed epoch analyses.

In Fig. 17 the results of superposed epoch analyses are separately shown for disturbances during different seasons (summer, winter, equinox). Whereas the mean geomagnetic disturbances are very similar in all three seasons (the maximum Ap index is in all cases greater than 100), the mean ionospheric effect is quite different. During summer and equinox conditions the main ionospheric effect is clearly negative with minimum values below $\mathrm{AI}=-40$, whereas in winter the negative effect is markedly smaller (lowest values near $\mathrm{AI}=-20$ ). This is in line with seasonal distribution of negative and positive phases of geomagnetic storms at European higher middle latitudes (e.g. Buresova et al., 2010).

\section{Discussion}

The proposed method of the determination of the reference value $f o \mathrm{~F}^{\text {ref }}$ in Eq. (1) has the advantage that some bias in the AI estimation caused by the seasonal $f o \mathrm{~F} 2$ variation can be eliminated. Nevertheless also with the old method (reference value estimated from the median value of the foregoing
27 or 30 days) reasonable results can be obtained as demonstrated by Bremer et al. (2006).

In this paper hourly $f_{o} \mathrm{~F} 2$ data of one mid-latitude station have been used. It is of course possible without any problems to estimate AI data also for other stations and for data with other measuring intervals (e.g. data every 5 min or every 15 min).

As mentioned above, also from $\mathrm{M}(3000) \mathrm{F} 2$ data ionospheric activity values can be derived by the same procedure as described in Sect. 2. Such data contain information about ionospheric height changes. However, these data are not so sensitive for the description of the ionospheric variability than those derived from $f o \mathrm{~F} 2$ observations.

As shown in Sects. 3.1 and 3.2 there are no mean diurnal and seasonal variations of the AI data (see Figs. 5, 6, 8). This is an important requirement to get homogenous data series of the ionospheric activity index AI. Seasonal variations can, however, been created if the reference values $f o \mathrm{~F}_{2}{ }^{\text {ref }}$ are erroneous (one example is shown in the middle part of Fig. 3).

The monthly median values of AI (see lower parts of Fig. 12) and the corresponding yearly mean values (see Fig. 7) depend significantly on solar activity. But there is no significant correlation with monthly or yearly averaged geomagnetic Ap indices. The calculated partial correlation coefficient between yearly averaged values of AI and F107 is markedly stronger $(r(\mathrm{AI}, \mathrm{F} 107 . \mathrm{Ap})=0.85)$ than by use of monthly median values $(r(\mathrm{AI}, \mathrm{F} 107 . \mathrm{Ap})=0.24)$. This feature is caused by the fact that ionospheric short term variations which do not depend on solar activity are better removed in yearly mean values of AI than in monthly median values. The detected changes of the mean AI data due to varying solar radiation are, however, only small (in general no more than $\pm 1 \%$ ) caused by the fact that both data sets, the current $f_{o} \mathrm{~F} 2$ values and the corresponding reference data $f o F^{\text {ref }}$, should vary in the same direction if the solar activity slowly changes.

The variability parameters of AI, the quartile difference (black curve in part (a) in Fig. 9), the standard deviation (red curve in part (a) in Fig. 9), and the cumulative occurrence rate COR for different thresholds (Fig. 10), are characterized by a marked seasonal variation with maxima at equinoxes, a pronounced minimum in summer and relative large values in winter. Main parts of this seasonal variation should be connected with the seasonal variation of the geomagnetic activity (see part (d) of Fig. 9 and left part of Fig. 11). The equinoctial maxima of the AI variability are most pronounced during high solar activity (upper part of Fig. 10) and should mainly be caused by the geomagnetic activity changes (see left part of Fig. 11 and strong correlation between dq as well as STD and Ap in parts concerning equinox conditions in Fig. 13). As the geomagnetic activity in summer and winter is comparable, the enhanced AI variability in winter may additionally be caused by stronger atmospheric variability due to atmospheric wave phenomena. 
The significant connection between the cumulative occurrence rates COR in Fig. 14 and the geomagnetic activity confirms the strong influence of the geomagnetic activity on the variability of the AI values. This influence is slightly stronger for negative AI values than for positive AI. This phenomenon may be caused by the fact that during ionospheric storms in middle latitudes mainly negative responses in AI can be detected (see Figs. 15-17).

The most remarkable ionospheric storm effects in midlatitudes can be observed in summer and equinoxes with distinct negative phases (see Fig. 17) whereas in winter only a small negative effect can be detected. This phenomenon is in general agreement with current ionospheric storm theories (Prölss, 2005; Hargreaves, 1992). In summer the equatorward-directed thermospheric wind transports atmospheric density changes from polar latitudes (increased ratio of molecular to atomic constituents) and creates in midlatitudes negative storm effects. In winter, however, the poleward-directed thermospheric wind prevents the propagation of such density changes to the equator at least partly. This effect depends on the amount of the auroral disturbance and the strength of the thermospheric wind. Therefore, positive and negative effects can be observed at mid-latitudes in winter.

\section{Conclusions and outlook}

The main results can be concluded with the following points:

- A modified ionospheric index AI has been derived by use of a new reference value calculated by a regression analysis of the $f o \mathrm{~F} 2$ values of the preceding 27 days at the same hour as the current $f o \mathrm{~F} 2$ value.

- The new index has no distinct diurnal and seasonal variation. This is the necessary precondition for the creation of homogeneous AI data series.

- AI data can be estimated for other stations, other time intervals, or other ionosonde parameters (e.g. M(3000)F2) without any problems.

- Monthly median and yearly mean values of AI do not depend on geomagnetic activity but depend on the solar activity level. However, the solar influence on the AI values is only small (not more than about $\pm 1 \%$ ).
- The variability of the AI values significantly depends on the geomagnetic activity, most clearly during equinoxes but also significant during summer and winter.

- Ionospheric storms in mid-latitudes are characterized during summer and equinox conditions by markedly reduced AI values. During winter the mean negative response is markedly smaller. These results are in general agreement with current storm theories.

Acknowledgements. The authors thank Jan Laštovicka and an anonymous referee for their helpful comments and suggestions.

Topical Editor K. Kauristie thanks J. Laštovicka and another anonymous referee for their help in evaluating this paper.

\section{References}

Belehaki, A., Cander, Lj. R., Zolesi, B., Bremer, J., Juren, C., Stanislawska, I., Dialetis, D., and Hatzopoulos, M.: DIAS Project: The establishment of a European digital upper atmosphere server, J. Atmos. Solar-Terr. Phys., 67, 1092-1099, doi:10.1016/j.jastp.2005.02.021, 2005.

Bremer, J., Cander, Lj. R., Mielich, J., and Stamper, R.: Derivation and test of ionospheric activity indices from real-time ionosonde observations in the European region, J. Atmos. Solar-Terr. Phys., 68, 2075-2090, doi:10.1016/j.jastp.2006.07.003, 2006.

Buresova, D., Mc Kinnell, L.-A., Sinderalova, T., and De La Morena, B. A.: Evaluation of the STORM model storm-time corrections for middle latitudes, Adv. Space Res., 46, 1039-1046, 2010.

Hargreaves, J. K.: The solar-terrestrial environment, Cambridge Atmospheric and Space Science Series, 5, Cambridge University Press, 1992.

Minnis, C. M.: A new index of solar activity based on ionospheric measurements, J. Atmos. Terr. Phys., 7, 310-321, 1955.

Minnis, C. M. and Bazzard, G. H.: Some indices of solar activity based on ionospheric and radio noise measurements, J. Atmos. Terr. Phys., 14, 213-228, 1959.

Minnis, C. M. and Bazzard, G. H.: A monthly ionospheric index of solar activity based on F2-layer ionization at eleven stations, J. Atmos. Terr. Phys., 18, 279-305, 1960.

Prölss, G. W.: Space weather effects in the upper atmosphere: Low and middle latitudes, in: Space weather, Lect. Notes Phys. 656, 193-214, edited by: Scherer, K., Fichtner, H., Heber, B., and Mall, U., Springer, Berlin, Heidelberg, 2005.

Taubenheim, J.: Statistische Auswertung geophysikalischer und meteorologischer Daten, Akad. Verlagsgesellschaft Geest \& Portig K. G., Leipzig, 1969. 